\title{
Anton-Babinski syndrome as a rare complication of chronic bilateral subdural hematomas
}

\author{
D. Adam ${ }^{1,2}$, D. Iftimie ${ }^{1}$, Cristiana Moisescu ${ }^{1}$, Gina Burduşa ${ }^{1}$ \\ ${ }^{1}$ Department of Neurosurgery, „Saint Pantelimon” Clinical Emergency Hospital, Bucharest \\ 2"Carol Davila" University of Medicine and Pharmacy, Bucharest, ROMANIA
}

\begin{abstract}
Clinical presentation: We report the case of a 68 -year-old patient that presented at our department 6 weeks after a mild traumatic brain injury with confusion and drowsiness. Emergency head CT scan revealed chronic bilateral subdural hematomas with no midline shift. His neurological status quickly deteriorated after admission (GCS of 9 points) and he was operated immediately. Although surgery was uneventful and his neurological status markedly improved, in the first postoperative day he developed blindness with visual anosognosia and visual confabulations, suggestive for Anton-Babinski syndrome. Emergency head MRI performed on the same day was normal but a repeat head MRI in the seventh postoperative day revealed bilateral occipital ischemia, thus explaining his symptoms. The patient became aware of his deficit two weeks after surgery, but was surprisingly ambivalent regarding it. Conclusion: Cortical blindness (sometimes presenting in the context of Anton-Babinski syndrome) is a rare complication of chronic bilateral subdural hematomas, a rather common neurosurgical pathology. Although it can be overlooked on the initial neurological examination, it should be taken into account, prompting emergency surgical treatment in order to prevent permanent visual deficits.

Key words: chronic bilateral subdural hematoma, visual anosognosia, visual confabulations
\end{abstract}

\section{Clinical presentation}

A 68-year-old patient, without significant medical history, suffered a mild traumatic brain injury secondary to a car accident, presenting no immediate symptoms at the time, nor for more than a month posttraumatic. Also, a CT scan was performed on the day of the accident, which was interpreted as normal. His family denied any other subsequent significant head trauma.

He was admitted in our Department 6 weeks after trauma, presenting confusion and drowsiness, symptoms that began six days prior to admission with progressive worsening. He was uncooperative, thus making a thorough neurological examination 
difficult and seemed to present no motor or cranial nerve deficits at the time. His GCS score at admission was noted as 13 by the attending physician.

Emergency head CT scan revealed chronic bilateral subdural hematomas (separated type according to the Nakaguchi classification1) with a maximal thickness of $1,6 \mathrm{~cm}$ on the left side and $1,3 \mathrm{~cm}$ on the right with no midline shift (Figure 1).

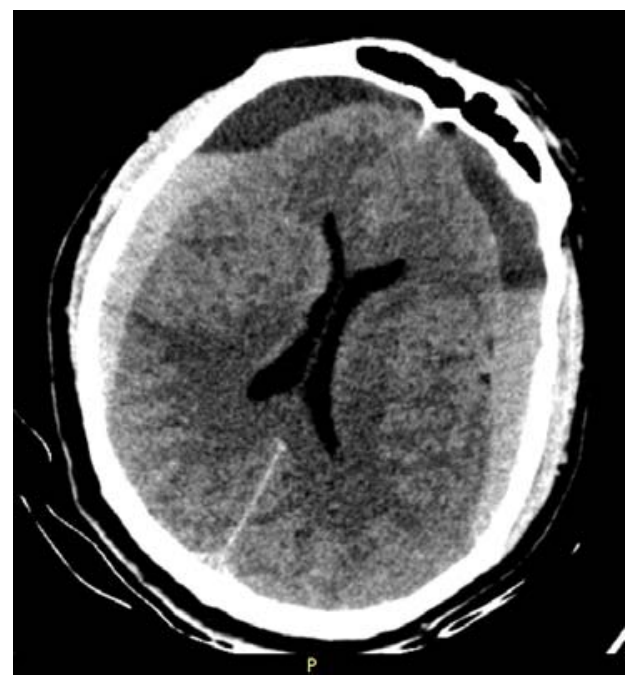

Figure 1 - Preoperative emergency head CT revealing bilateral chronic subdural hematomas with no midline shift

His neurological status rapidly deteriorated, with a GCS score of 9 several hours after admission. Emergency evacuation of bilateral hematomas was performed with fronto-parietal craniotomies. The operation was uneventful.

In the first postoperative day, his neurological status markedly improved, (GCS score of 14 points) but presented signs of visual disturbances which were not obvious at admission. Blindness with visual anosognosia and visual confabulations were noted, features suggestive for Anton-Babinski syndrome. Ophthalmological examination revealed no abnormalities and raised the suspicion of cortical blindness. Emergency MRI performed on the first postoperative day revealed no ischemic lesions (Figure 2).
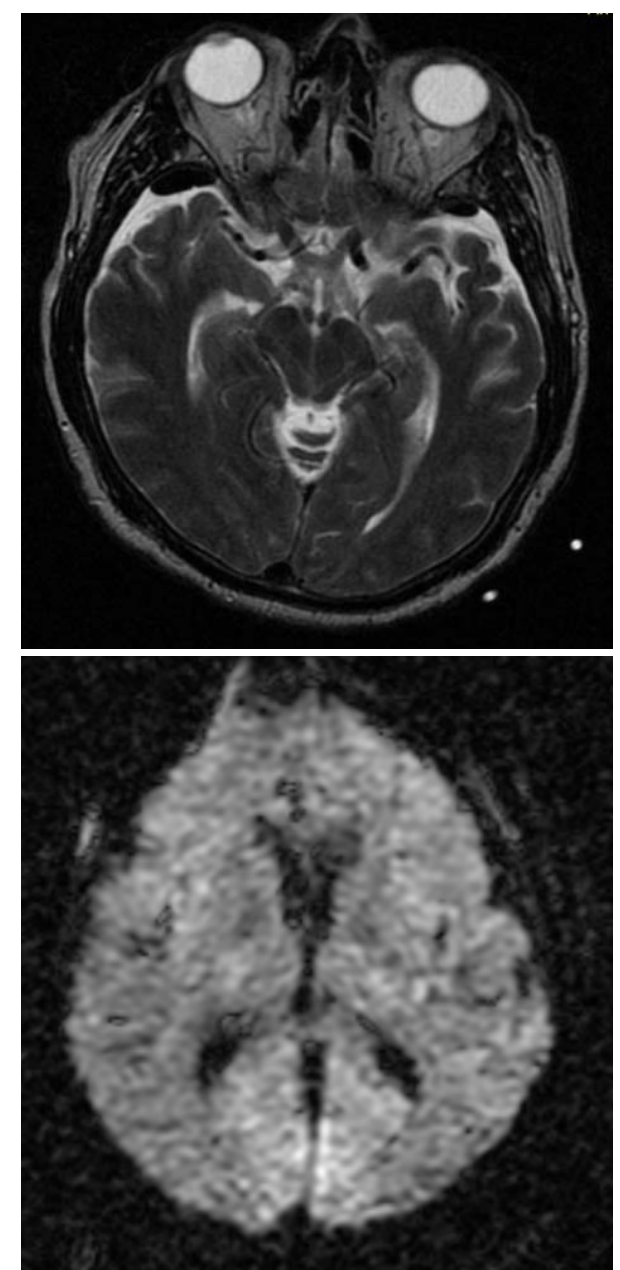

Figure 2 - Postoperative day 1 emergency head MRI with a normal postoperative appearance (left: T2 weighted image, right: DWI) 
Over the next few days, the patient presented no improvement, and remained blind. However, about two weeks after surgery he became permanently aware of his visual loss and his visual confabulations disappeared. The patient did not appear to be emotionally affected by this deficit and surprisingly remained in good spirits throughout his admission in our Department.

In the seventh postoperative day, an angioMRI with contrast was performed in an attempt to objectify the cause of the patient's blindness, that revealed bilateral occipital ischemia (Figure 3).
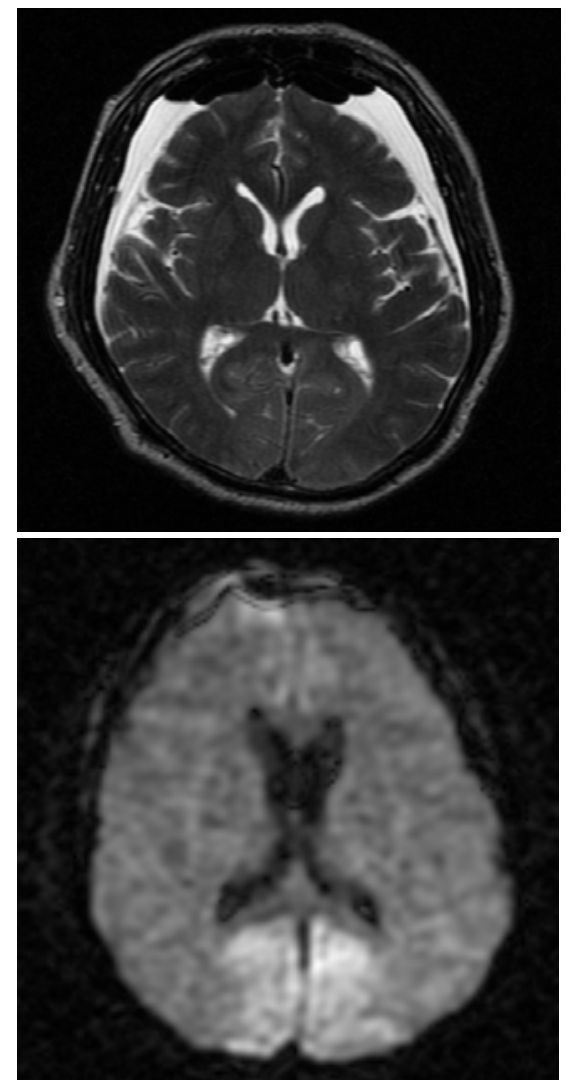

Figure 3 - Postoperative day 7 head MRI revealing bilateral occipital ischemic lesions (left: T2, right: DWI)
He was transferred to a Rehabilitation Department, his visual deficit remaining complete at a later follow-up.

\section{Discussion}

Montaigne (1533-1592) was probably the first to describe in one of his books a case of Anton-Babinski syndrome, describing a nobleman who did not believe he was blind despite obvious signs. Gabriel Anton (18581933) was the first to describe patients with blindness and deafness which were not aware of their deficit. Babinski was the one that later named this sign anosognosia. (2)

Blindness secondary to bilateral chronic subdural hematoma is a rare complication thus very few cases have been discussed in the literature. $(3,4)$ The majority of these cases presented progressive visual loss as a result of posterior cerebral artery infarction secondary to tentorial herniation. According to Keane's report, despite surgical evacuation, these patients were either left blind or with almost complete visual loss.

In 2008, Kabir reported a case of sudden blindness as a clinical manifestation of a chronic subdural hematoma. (5) In his case, following prompt surgical decompression, the patient's visual acuity returned to almost normal. However, in this case, the sudden blindness was proved to be caused by severe acute papilloedema, with no evidence of posterior cerebral artery territory ischemia, representing the only reported case of reversible blindness after chronic subdural hematoma evacuation.

In 2017, Balasubramanian et al (6) reported the case of a male patient who, after evacuation 
of a chronic bilateral subdural hematoma, developed bilateral posterior cerebral artery infarction and blindness. Based on a literature review, only four cases of blindness following chronic bilateral subdural hematoma evacuation are reported and all patients suffered permanent visual loss. The exact etiopathogenesis and mechanism of this rare complication are not completely understood.

We presented the case of a male patient with chronic bilateral subdural hematomas and postoperative permanent blindness as a result of bilateral occipital lobe ischemia. However, the case that we reported had a particularity: in addition to the cortical blindness, the patient also presented anosognosia with visual confabulation (characteristic for Anton Babinski syndrome), symptoms which were relevant only in the postoperative period. The Anton Babinski syndrome could have been present hours before the operation but it was not objectified as the patient was uncooperative at the time of admission, making a proper neurological examination difficult. Imaging evidence of bilateral occipital lobe ischemia was obtained in the seventh postoperative day.

Based on a review of the literature, it is difficult to establish awareness of blindness in patients with Anton-Babinski syndrome. Nevertheless, in one report, a patient with Anton- Babinski syndrome was aware of blindness within 2 weeks, without presenting any vision improvement. (7) In our case, the patient began to be aware of his visual loss approximately two weeks from the onset. However, he remained oddly optimistic given the circumstance. When asked every morning how he was feeling, he used to answer nonchalantly: „I cannot see but other than that, I am very well".

As far as we know, it would seem that this is the first case of chronic bilateral subdural hematomas associated with postoperative Anton-Babinski syndrome reported in the literature.

\section{Conclusion}

Cortical blindness (sometimes presenting in the context of Anton-Babinski syndrome) is a rare complication of chronic bilateral subdural hematomas, a rather common neurosurgical pathology. Although it can be overlooked on the initial neurological examination, it should be taken into account, prompting emergency surgical treatment in order to prevent permanent visual deficits.

\section{References}

1. Nakaguchi H, Tanishima T, Yoshimasu N. Factors in the natural history of chronic subdural hematomas that influence their postoperative recurrence. J Neurosurg. 2001;95(2):256-262. doi:10.3171/jns.2001.95.2.0256

2. Maddula M, Lutton S, Keegan B. Anton's syndrome due to cerebrovascular disease: a case report. J Med Case Rep. 2009;3:9028. doi:10.1186/1752-1947-00030000009028

3. Keane JR. Blindness following tentorial herniation. Ann Neurol. 1980;8(2):186-190. doi:10.1002/ana.410080209

4. Soza M, Tagle P, Kirkham T, Court J. Bilateral homonymous hemianopia with sparing of central vision after subdural hematoma. Can J Neurol Sci. 1987;14(2):153-155.

http://www.ncbi.nlm.nih.gov/pubmed/3607617. Accessed August 22, 2018.

5. Kabir S, Kamat A, Carroll T. Sudden Onset Blindness as a Presenting Feature of Chronic Subdural Haematoma: $\begin{array}{llll}\text { Case } & \text { Report. } & \text { Vol } & \end{array}$ 
https://print.ispub.com/api/0/ispub-article/13546.

Accessed August 22, 2018.

6. Chitra Balasubramanian S, Adimoolam S, Shunmugam S. Bilateral Chronic Subdural Hematoma and Blindness: Bilateral Infarction to the Posterior Cerebral Artery following Evacuation of Bilateral Chronic Subdural
Hematoma - A Rare Case Report and Review of Literature. Indian J Neurotrauma. 2017;14.

7. Chen J-J, Chang H-F, Hsu Y-C, Chen D-L. AntonBabinski syndrome in an old patient: a case report and literature review. Psychogeriatrics. 2015;15(1):58-61. doi:10.1111/psyg. 12064 